\title{
THREE-DIMENSIONAL COORDINATION NUMBER FROM TWO-DIMENSIONAL MEASUREMENTS: A NEW METHOD
}

\author{
By Richard B. AlLeY \\ (Geophysical and Polar Research Center, Department of Geology and Geophysics, \\ University of Wisconsin - Madison, Madison, Wisconsin 53706, U.S.A.)
}

\begin{abstract}
The average three-dimensional coordination number, $n_{3}$, is an important measure of firn structure. The value of $n_{3}$ can be estimated from $n_{2}$, the average measured two-dimensional coordination number, and from a function, $r$, that depends only on the ratio of average bond radius to grain radius in the sample. This method is easy to apply and does not require the use of unknown shape factors or tunable parameters.
\end{abstract}

RÉSUMÉ. Nombre coordonné à trois dimensions à partir de mesures bi-dimentionnelles une nouvelle méthode. La moyenne des coordonnées à trois dimensions, $n_{3}$, est une mesure importante de la structure du névé. La valeur de $n_{3}$ peut être estimée à partir de $n_{2}$, nombre moyen des mesures à deux dimensions et à partir d'une fonction $\mathrm{r}$, qui dépend

\section{INTRODUCTION}

Some physical properties of any granular material depend on the average coordination number of grains in the material (Gubler, 1978) but coordination number has proven a difficult quantity to measure. (Here, coordination number is defined as the number of grains in direct contact with a given grain; the surface of contact between grains is a grain bond.) The most accurate way to measure coordination number is by careful examination of closely spaced serial sections of a material. This method is so time-consuming, however, that it is generally impractical. It is faster but less accurate to estimate coordination numbers from measurements taken on a single section plane. Several methods have been proposed for doing this but all suffer from serious flaws. Here, we briefly review these previously published methods and then present a new method for estimating three-dimensional coordination number from measurements on a single plane of section.

\section{SYMBOLS USED}

$a$

$A$

$A_{\mathrm{m}}$

\section{$C^{\prime}$}

\section{Average bond area}

Average cross-sectional area of grains

Average cross-sectional area of grains on plane of section

Shape factor

Harmonic mean of bond lengths on plane of section

Fraction of bonds intersected by a random cut of a grain

$F_{1}$

Value of $F$ for cut near center of grain

$F_{2}$

Value of $F$ for cut near edge of grain

Tunable parameter in model of Gubler (1978)

uniquement du rapport entre le rayon moyen de voisinage et de celui du grain de l'échantillon. Cette méthode est d'un emploi facile et ne nécessite pas l'utilisation de facteurs de forme inconnus ni de paramètres ajustables.

Zusammenfassung. Dreidimensionale Zuordnungszahl aus zweidimensionalen Messungen: Ein neues Verfahren. Die mittlere dreidimensionale Zuordnungszahl $n_{3}$ ist ein wichtiges Mass für die Firnstruktur. Der Wert von $n_{3}$ kann aus $n_{2}$, der mittleren gemessenen zweidimensionalen Zuordnungszahl, und aus einer Funktion $\mathrm{I}$, die nur vom Verhältnis des mittleren Bindungsradius zum Kornradius abhängt, abgeschätzt werden. Dieses Verfahren ist leicht anzuwenden und erfordert keine Kenntnis von Formfaktoren oder Abstimmungsparametern.

$n_{2}$

$f_{2}\left(n_{2 j}\right) \quad \begin{gathered}\text { Distribution of coordination numbers on plane of } \\ \text { section }\end{gathered}$

$n_{3}$

$f_{3}\left(n_{3 j}\right) \quad$ Distribution of three-dimensional coordination numbers

$n_{3}{ }^{\prime} \quad$ Value of $n_{3}$ calculated following Alley and others (1982)

$n_{3}$ * Value of $n_{3}$ calculated following Kry (1975)

$N_{\mathrm{A}} \quad$ Number of grains per unit area on plane of section

$N_{\mathrm{Ab}} \quad$ Number of bonds per unit area on plane of section

$N_{\text {Lb }} \quad$ Number of bonds per unit length of test line on plane of section

$N_{\text {Lf }} \quad$ Number of free surfaces (ice-air contacts) per unit length of test line on plane of section

$N_{\mathrm{v}} \quad$ Number of grains per unit volume

$N_{\mathrm{vb}} \quad$ Number of bonds per unit volume

p Probability of a cut intersecting a bond in model of Gubler (1978)

$r \quad$ Average radius of grain bonds section

$R$

$R_{\mathrm{m}}$
Average radius of grains

Average of radii of grains seen on a plane of section 
$r^{\prime} \quad$ Average radius of grain bonds calculated from average bond area

$R^{\prime}$

Average radius of grains calculated from average grain area

\section{$\boldsymbol{z}$}

$z^{\prime}$

$z^{\prime \prime}$

$\alpha$

$\alpha^{\prime}$

B

r

$r_{\text {ins }}$

$\Gamma_{\tan }$

\section{PREVIOUS METHODS}

All of the methods for estimating three-dimensional coordination number, $n_{3}$, from measurements on a planar cross-section involve the use of certain simple counting measurements and certain assumptions regarding the size, derived quantities used in such methods are given above. The counting measurements and simple derived quantities above $\left(A_{\mathrm{m}}, B, E, n_{2}, f_{2}\left(n_{2 j}\right), N_{\mathrm{A}}, N_{\mathrm{Ab}}, N_{\mathrm{Lb}}, N_{\mathrm{Lf}}\right)$ require only that analysis be conducted on a random plane in an isotropic material or that averages be computed over all directions in an anisotropic material. All other quantities given above depend on assumptions regarding geometry in the material, and these assumptions generally cannot be tested rigorously. It is thus important that the method selected for calculating $n_{3}$ be insensitive to deviations from assumed geometry. Further discussion of both basic measurements and derived quantities can be found in a number of sources, including Underwood (1970), Kry (1975), and Alley and others (1982). The measurement of $n_{2}$ and its use as a qualitative indicator of firn densification has been discussed by Fuchs (1959) and Ebinuma and Maeno (1985). We will present only a sketchy development of this material here.

Calculation of $n_{3}$ for planar, circular grain bonds of uniform size between mono-sized spheres was considered by Underwood (1970, p. 102). He showed that

$$
\begin{gathered}
n_{3}=\frac{2 N_{\mathrm{vb}}}{N_{\mathrm{v}}} \\
N_{\mathrm{vb}}=\frac{2\left(N_{\mathrm{Ab}}\right)^{2}}{\pi N_{\mathrm{Lb}}}
\end{gathered}
$$

where $N_{\mathrm{Lb}}$ is the number of intersections per unit length between randomly oriented test lines and grain bonds on the plane of section, $N_{\mathrm{Ab}}$ is the number of grain bonds per unit area intersected by the plane of section, $N_{\mathrm{vb}}$ is the number of grain bonds per unit volume, and $N_{\mathrm{v}}$ is the number of grains per unit volume. The quantity $N_{\mathrm{v}}$ is strongly dependent on the grain shape. For spherical grains, Underwood (1970, p. 96) showed that

$$
N_{\mathrm{v}}=\frac{N_{\mathrm{A}}}{2 R}
$$

where $N_{\mathrm{A}}$ is the number of grains per unit area intersected by the plane of section and $R$ is the average grain radius. The variation of $N_{\mathrm{y}}$ with grain shape has been discussed by DeHoff and Rhines (1961), who showed that relatively small shape variations from sphericity can lead to errors in $N_{\mathrm{v}}$ in excess of $100 \%$ if Equation (2) is used. Although DeHoff and Rhines (1961) derived equations equivalent to Equation (2) for a variety of shapes including prolate and oblate spheroids of arbitrary axial ratio, they assumed that all grains in a material have the same shape. This requirement of shape constancy is not met in many real materials, so that estimates of $N_{\mathrm{v}}$ should be considered inaccurate. We thus follow Kry (1975) in considering that any estimate of $n_{3}$ based on $N_{\mathrm{v}}$ should not be used to draw quantitative conclusions.

The assumption of uniform bond size used in Equation (1) was relaxed by Fullman (1953) and Kry (1975), who showed that

$$
\begin{aligned}
n_{3} & =\frac{2 N_{\mathrm{vb}}}{N_{\mathrm{v}}} \\
N_{\mathrm{vb}} & =\frac{8 N_{\mathrm{Ab}} E}{\pi^{2}}
\end{aligned}
$$

where $E$ is the harmonic mean of the lengths of intersections of bonds with the plane of section. Again, this method yields excellent results if and only if $N_{\mathrm{v}}$ can be evaluated accurately. Considering the uncertainty in $N_{\mathrm{v}}, \mathrm{Kry}$ (1975) went so far as to suggest that this method may yield little better than order-of-magnitude estimates of $n_{3}$. Natural variation of $n_{3}$ is little more than an order of magnitude, so this is not too useful.

A slightly different approach was adopted by Alley and others (1982), who estimated that

$$
\begin{aligned}
n_{3} & =\frac{6 C^{\prime} A_{\mathrm{m}} \beta}{a} \\
B & =\frac{2 N_{\mathrm{Lb}}}{2 N_{\mathrm{Lb}}+N_{\mathrm{Lf}}} \\
a & =\frac{2 N_{\mathrm{Lb}}}{N_{\mathrm{vb}}}
\end{aligned}
$$

where $C^{\prime}$ is a shape factor (discussed below), $A_{\mathrm{m}}$ is the average area of grains observed on a plane of section, $B$ is the fraction of grain surface occupied by bonds, $N_{\mathrm{Lf}}$ and $N_{\text {Lb }}$ are respectively the number of intersections per unit length of randomly oriented test lines with ice-air surfaces and with grain bonds, $a$ is the average area of bonds, and $N_{\text {vb }}$ is given in Equation (3). (Note that the equation for $B$ in Alley and others (1982, p. 9) is in error; the correct expression is given above.) The estimate of $a$ is from Fullman (1953) and assumes circular, planar bonds; this assumption of bond shape was tested by Kry (1975) and Alley (paper in preparation), and seems to be an accurate approximation. The factor $\left(6 C^{\prime} A_{\mathrm{m}}\right)$ in Equations (4) is the average surface area per grain, and the shape factor $C^{\prime}$ corrects for deviations of grains from spherical form and for grain-size distributions. The shape factor was taken to be identically 1 by Alley and others (1982) but it can vary significantly with grain shape (Underwood, 1970, p. 90-93; Table I). The shape factor is not known a priori for any real material and is thus the major source of error in this method. This method may be more accurate than those relying on $N_{\mathrm{v}}$ but less accurate than we would like. 
TABLE I. VALUES OF SHAPE FACTOR $C^{\prime}$ IN EQUATION (4), FROM UNDERWOOD (1970, p. 90-93)

Figure

Oblate spheroid, axial ratio $=0.5$

Sphere

Pentagonal dodecahedron

Truncated octahedron (tetrakaidecahedron)

Prolate spheroid, axial ratio $=0.5$

Hemisphere

Rhombic dodecahedron

Cube

An interesting approach developed by Gubler (1978) for study of seasonal snow leads to an estimate of the distribution of coordination numbers $f_{3}\left(n_{3}\right)$. It was shown by Gubler (1978) that any assumed distribution $f_{3}\left(n_{3 j}\right)$ can be used to predict a distribution function of coordination numbers in a plane of section, $f_{2}\left(n_{2}, p, i\right)$, that depends on a tunable parameter, $i$, and on the probability, $p$, that a random cut through a single grain with one bond will intersect that bond. The probability, $p$, depends on average grain radius, $R$, average bond radius, $r$, the number of grains per unit volume, $N_{\mathrm{v}}$, an empirically evaluated constant, and a shape factor that must be estimated. To calculate three-dimensional coordination, $p$ is calculated, trial values of $f_{3}\left(n_{3}\right)$ and $i$ are selected, and the two-dimensional distribution function $f_{2}\left(n_{2 j}, p, i\right)$ consistent with the trial values is calculated. Then $f_{3}\left(n_{3 j}\right)$ and $i$ are adjusted until the predicted $f_{2}\left(n_{2}, p, i\right)$ matches the observed two-dimensional distribution $f_{2}\left(n_{2}\right)$ as closely as possible. The trial distribution $f_{3}\left(n_{3 j}\right)$ that produces the closest match is then the best estimate of the actual threedimensional distribution of coordination numbers. This method requires that $(r / R) \ll 1$, which is realized in the snow studied by Gubler (1978) but not in most firn (Alley and others, 1982; paper in preparation by R.B. Alley). Although this method may prove valuable in the study of seasonal snow, the use of a tunable parameter, a shape factor, an empirical constant, and the uncertain quantity $N_{\mathrm{v}}$, and the requirement of small bonds, render it suspect for application in firn. The use of observed two-dimensional coordination numbers to estimate three-dimensional coordination is an excellent idea, however, and we adopt it in developing our new model.

\section{MODEL}

Stated briefly, we have developed a transfer function from the average two-dimensional coordination number on a plane of section, $n_{2}$, to the average three-dimensional coordination number in the material, $n_{3}$, based on the average probability that a plane of section will intersect a circular, planar bond on the surface of a spherical grain. No solution is available for the exact geometry of bonds on grains, so we construct limiting cases and choose their average value for our transfer function. The transfer function is

$$
\begin{aligned}
n_{3} & =\frac{n_{2}}{\mathrm{I}(\alpha)} \\
\alpha & =\frac{r}{R}
\end{aligned}
$$

where $\Gamma$, the average fraction of bonds intersected by a plane of section through a sphere, depends only on $\alpha$, the ratio of average bond radius, $r$, to average grain radius, $R$.

Consider a spherical grain of radius $R$ with a planar, circular grain bond of radius $r$. Clearly, for $r>0$ the grain
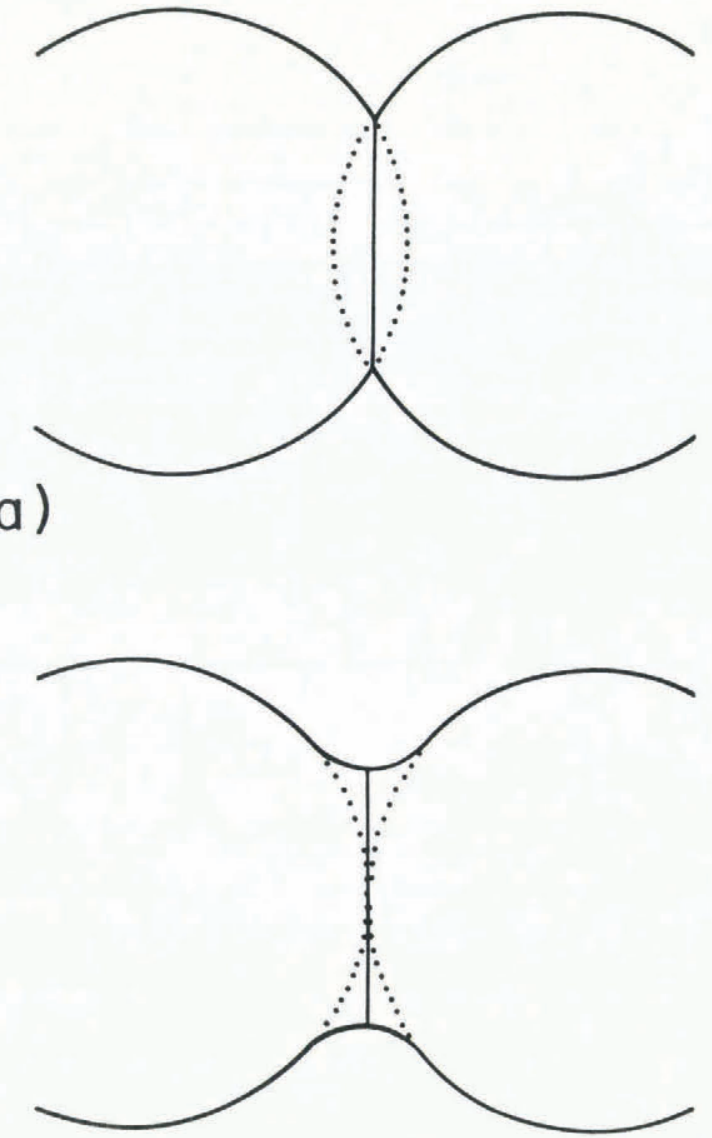

b)

Fig. 1. Limiting geometries for grain bonds. (a) Inscribed bonds. (b) Tangential bonds.

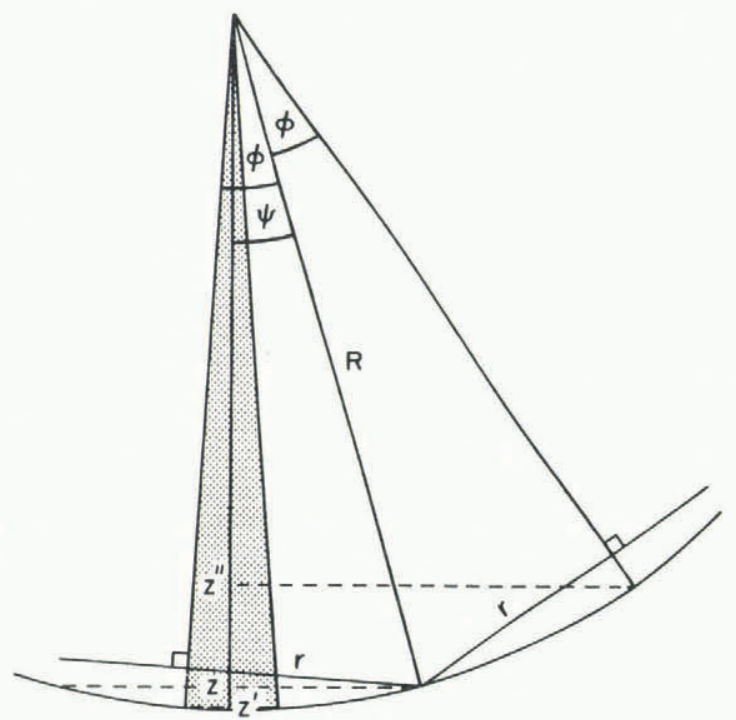

Fig. 2. Geometry for inscribed grain bonds, with plane of section near center of grain.

cannot be a true sphere. The actual geometry is limited by the cases of inscribed bonds (Fig. 1a) and tangential bonds (Fig. 1b). We model each case by calculating $r_{\text {ing }}$ for inscribed bonds and $\mathrm{r}_{\tan }$ for tangential bonds, and then take $I$ to be the average of the two.

We begin with the inscribed case, which is shown in detail in Figure 2. Bonds are assumed to be circles of radius $r$ distributed randomly over the grain. A section cut at position $z$ will intersect all bonds with centers falling between $z^{\prime}$ and $z^{\prime \prime}$ (see Fig. 2). The region between $z^{\prime}$ and $z^{\prime \prime}$ contains surface area of the sphere $2 \pi R\left(z^{\prime}-z^{\prime \prime}\right)$ out of 
total surface area $4 \pi R^{2}$; thus, the cut at $z$ intersects fraction $F_{1}$ of the total bonds on the grain, where

$$
F_{1}=\frac{2 \pi R\left(z^{\prime}-z^{\prime \prime}\right)}{4 \pi R^{2}} .
$$

(The spatial distribution on a grain of bonds of non-zero size cannot be truly random; however, we require only that, on average, fraction $F_{1}$ of the surface area of a grain contains fraction $F_{1}$ of the bond centers on that grain, which is realized if bonds lack a preferred orientation.)

From the geometry of Figure 2

$$
\begin{aligned}
& z^{\prime}=R \cos (\psi-\phi), \\
& z^{\prime \prime}=R \cos (\psi+\phi) .
\end{aligned}
$$

Equations (7) can be re-written in terms of sines and cosines of $\psi$ and $\phi$ using standard trigonometric identities, which can then be re-written in terms of $R, \alpha$, and $z$. This leads to

$$
\begin{aligned}
& z^{\prime}=z \sqrt{1-\alpha^{2}}+\alpha \sqrt{R^{2}-z^{2}}, \\
& z^{\prime \prime}=z \sqrt{1-\alpha^{2}}-\alpha \sqrt{R^{2}-z^{2}}, \\
& z^{\prime}-z^{\prime \prime}=2 \alpha \sqrt{R^{2}-z^{2}} .
\end{aligned}
$$

We have ignored one complication thus far, however. When $\psi<\phi$, a cut at $z$ will not intersect the grain at all if there is a grain bond centered between $(\psi-\phi)$ and $(\phi-$ $\psi)$, as shown in Figure 3. This region has area $2 \pi R(R-$ $\left.z^{\prime}\right)$. If a cut with $\psi<\phi$ intersects the grain, then there

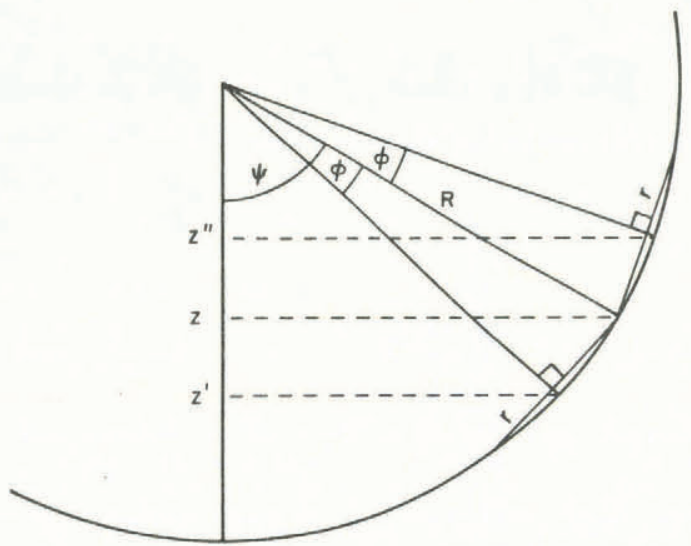

Fig. 3. Geometry for inscribed grain bonds, with plane of section near edge of grain. Shaded region lies between $(\phi-\psi)$ and $(\phi-\phi)$.

are no bonds centered in this region. In this case, the total area sampled by the cut is still given by $2 \pi R\left(z^{\prime}-z^{\prime \prime}\right)$, but the total area over which bonds can occur is $4 \pi R^{2}-2 \pi R(R$ $\left.-z^{\prime}\right)$. Thus, for $\psi<\phi$, a cut at $z$ samples $F_{2}$ of the total bonds, where

$$
F_{2}=\frac{2 \pi R\left(z^{\prime}-z^{\prime \prime}\right)}{4 \pi R^{2}-2 \pi R\left(R-z^{\prime}\right)}
$$

By direct geometry, we can show that when $\phi=\psi, z=$ $R \sqrt{1-\alpha^{2}}$. This allows us to write the fraction of bonds, $F$, intersected by a cut at $z$, as

$$
F=\left\{\begin{array}{l}
F_{2},-R \leqslant z \leqslant-R \sqrt{1-\alpha^{2}}, \\
F_{1},-R \sqrt{1-\alpha^{2}}<z \leqslant R \sqrt{1-\alpha^{2}}, \\
F_{2}, \quad R \sqrt{1-\alpha^{2}}<z \leqslant R .
\end{array}\right.
$$

Because of the spherical symmetry of the problem, the average fraction of bonds sampled by a cut through a grain, $\mathrm{r}_{\text {ins, }}$ is simply the average of $F$ over all $z$ along any diameter of the spherical grain. Thus

$$
\begin{gathered}
\mathrm{r}_{\mathrm{ins}}=\frac{1}{2 R} \int_{-R}^{R} F \mathrm{~d} z, \\
\mathrm{r}_{\mathrm{ins}}=\frac{1}{2 R}\left\{\int_{-R \sqrt{1-\alpha^{2}}}^{R \sqrt{1-\alpha^{2}}} \frac{\alpha}{R} \sqrt{R^{2}-z^{2}} \mathrm{~d} z+\right. \\
\left.+2 \int_{R \sqrt{1-\alpha^{2}}}^{R} \frac{2 \alpha \sqrt{R^{2}-z^{2}}}{z \sqrt{1-\alpha^{2}}+\alpha \sqrt{R^{2}-z^{2}}} \mathrm{~d} z\right\}
\end{gathered}
$$

The first integral in Equation (12) can be evaluated directly, and Equation (12) becomes

$$
\begin{aligned}
\mathrm{I}_{\mathrm{ins}} & =\alpha \sqrt{1-\alpha^{2}}+\sin ^{-1} \sqrt{1-\alpha^{2}}+ \\
& +\frac{1}{R} \int_{R \sqrt{1-\alpha^{2}}}^{R} \frac{2 \alpha \sqrt{R^{2}-z^{2}}}{R+z \sqrt{1-\alpha^{2}}+\alpha \sqrt{R^{2}-z^{2}}} \mathrm{~d} z .
\end{aligned}
$$

The remaining integral can be evaluated numerically without great difficulty and is a function of $\alpha$ only.

We should note here that the second integral in Equation (12) is a small correction term except at large $\alpha$. Had we ignored this term and evaluated the first integral from $-R$ to $R$, the result would have been

$$
\mathrm{r}_{\mathrm{ina}} \approx \frac{\pi \alpha}{4}
$$

Equations (13) and (14) differ by only $8 \%$ for $\alpha=1.0$, by only $0.2 \%$ for $\alpha=0.7$, and are identical to four significant figures for $\alpha<0.5$; thus, Equation (14) could be used in place of Equation (13) in most cases.

Next, consider the tangential case shown in Figure 4 . The tangential bond to a sphere of radius $R$ is equivalent to an inscribed bond in a sphere of radius $R \sqrt{1+\alpha^{2}}$. The tangential case with relative bond size $\alpha$ thus behaves like the inscribed case with bond size $\alpha^{\prime}$, where

$$
\alpha^{\prime}=\frac{\alpha}{\sqrt{1+\alpha^{2}}}
$$

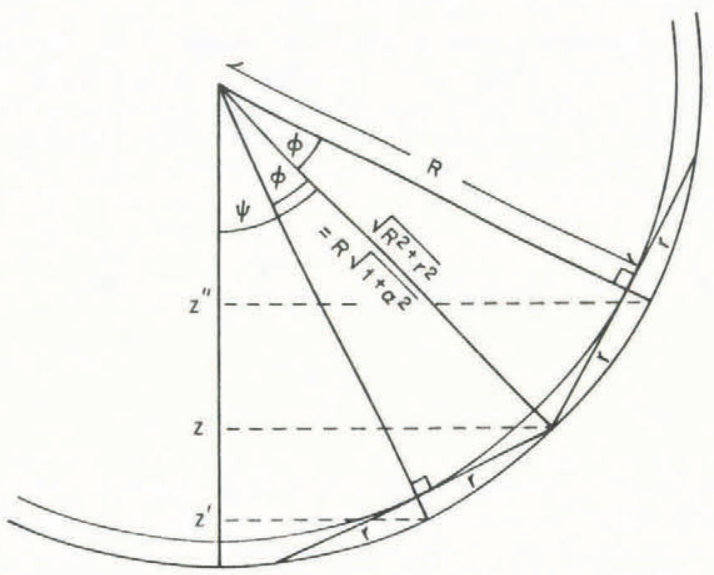

Fig. 4. Geometry for tangential grain bonds. 
and

$$
\Gamma_{\tan }(\alpha)=\Gamma_{\text {ins }}\left(\alpha^{\prime}\right) .
$$

The true $I$ for a given grain lies between $r_{\text {ins }}$ and $r_{\text {tan }}$, so we choose as the best estimate

$$
\Gamma(\alpha)=\frac{\Gamma_{\text {ins }}(\alpha)+r_{\tan }(\alpha)}{2} .
$$

Values of $\Gamma(\alpha)$ are listed in Table II, together with the relative difference between $r$ and $r_{\text {ins }}$ or $r_{\tan }$. Based on experience, we do not expect natural values of $\alpha$ to exceed 0.7 commonly, so Table II shows that the maximum uncertainty introduced by choosing $\Gamma$ rather than $\Gamma_{\text {ins }}$ or $\Gamma_{\text {tan }}$ is less than $10 \%$. In most cases, the error introduced by using I should be very small.

Equation (17) and Table II represent our best estimate

TABLE II. $\Gamma$ VERSUS $\propto$ FROM EQUATION (17), AND MAXIMUM RELATIVE ERROR IN I FROM ASSUMED SHAPE

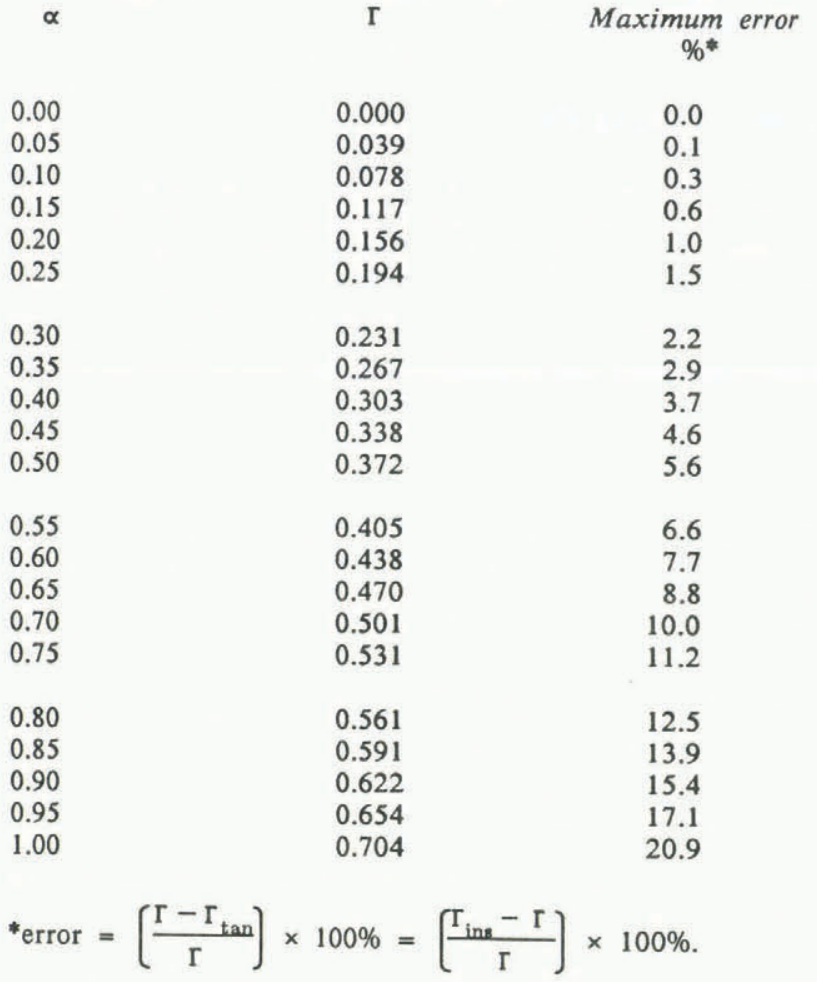

of $\Gamma$. If we use the approximation in Equation (14), then we can estimate $I$ as

$$
I \approx \frac{\pi \alpha}{4}\left[\frac{1+\left(1+\alpha^{2}\right)^{-1 / 2}}{2}\right] .
$$

This is an excellent approximation of Equation (17) except at large $\alpha$.

\section{CALCULATIONS}

This method requires that $r$ and $R$, the average radii of bonds and grains, be known so that $\alpha$ can be calculated. For circular bonds that may exhibit a bond-size distribution, Fullman (1953) showed that

$$
r=\frac{\pi}{4 E}
$$

where $E$ is the harmonic mean of the lengths of inter- sections of bonds with the plane of section. For mono-sized spherical grains, it is not difficult to show that

$$
R=\frac{4 R_{\mathrm{m}}}{\pi}
$$

where $R_{\mathrm{m}}$ is the average of individual radii of grains seen on the plane of section. This may be a good estimate for non-spherical grains of different sizes (Mendelson, 1969), although this is difficult to demonstrate rigorously. Methods for determining $R$ from measured intercept lengths were also discussed by Mendelson (1969). (We recognize the imprecision introduced by not treating explicitly the effect on $R$ and $\alpha$ of a distribution of grain shapes and sizes, but grain-size itself is not a well-defined quantity unless all grains have a specified shape (Underwood, 1970); we are continuing to investigate this problem.) Once $r$ and $R$ are known, then $\alpha$ is calculated from

$$
\alpha=\frac{r}{R} \text {. }
$$

In many cases, it is easier to calculate $\alpha$ from $r^{\prime}$ and $R^{\prime}$, which are calculated from average areas. For circular bonds of different sizes, Fullman (1953) showed that the average bond area, $a$, is given by

$$
a=\frac{\pi^{2} N_{\mathrm{Lb}}}{4 N_{\mathrm{Ab}} E} .
$$

The true average cross-sectional area of grains, $A$, is related in some fashion to the measured cross-sectional area on the plane of section, $A_{\mathrm{m}}$. For mono-sized spherical grains, $A$ is given exactly by

$$
A=\frac{3}{2} A_{\mathrm{m}}
$$

This is a slight overestimate of $\boldsymbol{A}$ for a sample consisting of spherical grains of different sizes and a slight underestimate of $A$ for mono-sized, non-spherical grains, and so should be a good estimate for non-spherical grains of different sizes (paper in preparation by R.B. Alley). Then

$$
\alpha \approx \sqrt{\frac{a}{A}}=\frac{r^{\prime}}{R^{\prime}} \text {. }
$$

Empirically, we find that Equations (24) and (21) differ by less than $5 \%$ in most cases, so the investigator should choose the more convenient.

\section{DISCUSSION}

Because of the near-impossibility of learning $n_{3}$ exactly in a real sintered material, we cannot provide a rigorous test of our new method for calculating $n_{3}$. As discussed below, however, several factors recommend our method, including its insensitivity to deviations from assumptions, its independence from shape factors or tunable parameters, its accuracy, and its ease of computation.

No real system will match exactly the geometry assumed in our derivation. A grain can deviate from a spherical form toward some other convex form or toward a non-convex form. In either case, however, the deviation will affect in a similar manner both the total surface area of the grain and the average surface sampled by a plane of section; thus, I will vary more slowly than any single measure of grain shape. As an extreme example, the value of $I$ for a thin disc of radius $R$ is only about $(4 / \pi)$ times $I$ for a sphere of radius $R$, if $r$ is held fixed.

As we discussed above, some other published methods of calculating $n_{3}$ rely on shape factors or tunable parameters that cannot be estimated well. Our new model does not require any of these and so is preferable. (Deviations from sphericity could be corrected by a shape factor but the slow variation of $\Gamma$ with grain shape allows us to set this shape factor to 1.)

A major objection to other methods of calculating $n_{3}$ is 
their reliance on quantities, particularly $N_{\mathrm{v}}$, that cannot be determined accurately. Our method depends on $n_{2}$ and on $\propto$ the latter does depend on a number of measured and calculated quantities. However, both experience and theory (Alley and others, 1982; paper in preparation by R.B. Alley) indicate that $\alpha$ (and thus $\mathrm{r}$ ) varies by a factor of 2 or less after a bonded structure is developed. Most variations in $n_{3}$ thus result from variations in $n_{2}$. The value of $n_{2}$ can be determined with considerable accuracy. In typical firn of density $0.55 \mathrm{Mg} \mathrm{m}^{-3}$ from "Upstream B" on the Siple Coast of West Antarctica, a $t$-test on a count of 100 grains typically yields $n_{2}=2.5 \pm 0.17$ with $90 \%$ confidence. Counting more grains would narrow the confidence interval further. We believe that the total accuracy of our method is better than $20 \%$, although we cannot demonstrate this rigorously. (For values of $\alpha$ less than 0.1 , difficulty in recognizing a contact and a large standard deviation on $n_{2}$ will decrease the accuracy.)

Finally, our new method allows easy computation. Although $r$ and $R$ can be determined only after substantial effort, they are frequently of interest in their own right. Once $r$ and $R$ (or $r^{\prime}$ and $R^{\prime}$ ) are known, our model requires only that $n_{2}$ be measured and I determined from Table II or Equation (18), and these substituted into Equation (5). The total time required for measurement and computation after $r$ and $R$ are known is typically $10-15$ min.

As an exercise, we compared $n_{3}$ calculated using our new method with $n_{3}^{\prime}$ calculated after Alley and others (1982) for 20 samples from "Upstream B" on the Siple Coast of West Antarctica. Values of $n_{3}$ are plotted in Figure 5. If we

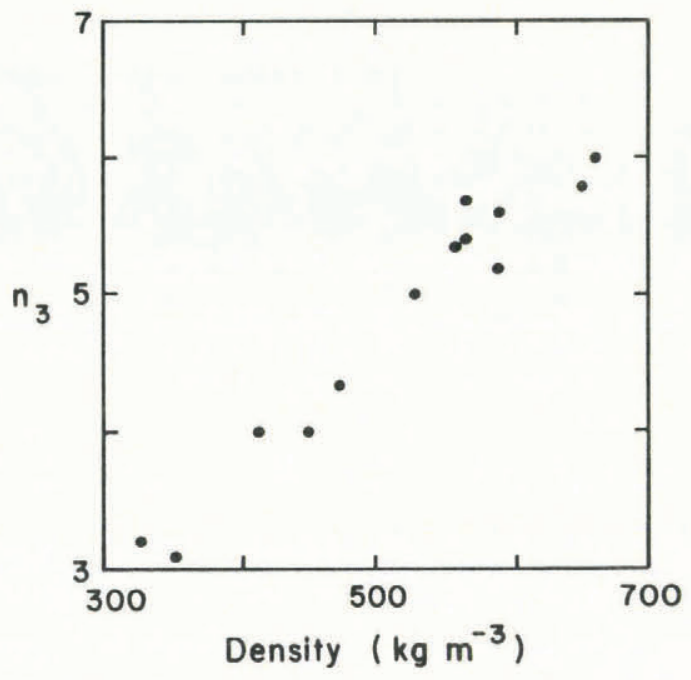

Fig. 5. Values of $n_{3}$ versus density for "Upstream $B$ " on the Siple Coast of West Antarctica. Values of $n_{3}$ for anisotropic firn were obtained by averaging values for horizontal and vertical sections from the same sample.

take $C^{\prime}=1$ following Alley and others (1982), then $n_{3}^{\prime}<$ $n_{3}$ in every case considered. If we assume that $n_{3}$ from our new method is exact and calculate $C^{\prime}$ for the samples, we obtain a mean value of $C^{\prime}=1.29$ with a standard deviation of 0.15 . Actual grains in shallow firn probably range from spheres to prolate spheroids and become more like truncated octahedra (tetrakaidecahedra) or dodecahedra with increasing depth; also, actual grains probably have some surface irregularities which would tend to increase $C^{\prime}$. In the light of these considerations, Table $I$ shows that $C^{\prime}=1.3$ is a reasonable value, which tends to lend credence to our model. We emphasize, however, that $C^{\prime}$ need not be the same in different samples and cannot be known a priori.
We also tested our new model against $n_{3}{ }^{*}$ from Fullman (1953) and Kry (1975), assuming spherical grains. Results show some variability but in general $n_{3}$ " is $10-20 \%$ less than $n_{3}$. Grains are not spherical in real firn but resemble prolate ellipsoids. Agreement between $n_{3}$ " and $n_{3}$ would be improved significantly if we assumed the grains to be prolate ellipsoids of axial ratio 0.9 (DeHoff and Rhines, 1961). This is a reasonable value based on observation but cannot be derived readily from measurements.

\section{CONCLUSIONS}

We have presented a new method for calculating $n_{3}$, the average three-dimensional coordination number in a granular material, from stereological measurements on a section plane. The model is computationally simple, accurate, insensitive to deviations from assumptions used in its derivation, and requires no shape factors or tunable parameters. Differences between results from our new model and previous models are explicable based on known weaknesses in the previous models. Thus, we believe that our model provides a useful way to estimate $n_{3}$. We now are using data on $n_{3}$ to study densification processes in firn (Alley and Bentley, in press).

\section{ACKNOWLEDGEMENTS}

Financial support for this work was provided by the U.S. National Science Foundation under grant DPP-8315777. We thank C.R. Bentley, J.F. Bolzan, J.H. Perepezko, H.F. Wang, and I.M. Whillans for helpful suggestions and A.N. Mares and S.H. Smith for manuscript preparation. This is contribution No. 450 of the Geophysical and Polar Research Center, University of Wisconsin-Madison.

\section{REFERENCES}

Alley, R.B., and Bentley, C.R. In press. Firn studies at Upstream B, West Antarctica. Antarctic Journal of the United States.

Alley, R.B., and others. 1982. Polar firn densification and grain growth, by R.B. Alley, J.F. Bolzan, and I.M. Whillans. Annals of Glaciology, Vol. 3, p. 7-11.

Dehoff, R.T., and Rhines, F.N. 1961. Determination of number of particles per unit volume from measurements made on random plane sections: the general cylinder and the ellipsoid. Transactions of the Metallurgical Society of $A I M E$, Vol. 221 , p. $975-82$.

Ebinuma, T., and Maeno, N. 1985. Experimental studies on densification and pressure-sintering of ice. Annals of Glaciology, Vol. 6, p. 83-86.

Fuchs, A. 1959. Some structural properties of Greenland snow. U.S. Snow, Ice, and Permafrost Research Establishment. Research Report 42.

Fullman, R.L. 1953. Measurement of particle sizes in opaque bodies. Transactions of the Metallurgical Society of $A I M E$, Vol. 197, p. 447-52.

Gubler, H. 1978. Determination of the mean number of bonds per snow grain and of the dependence of the tensile strength of snow on stereological parameters. Journal of Glaciology, Vol. 20, No. 83, p. 329-41.

Kry, P.R. 1975. Quantitative stereological analysis of grain bonds in snow. Journal of Glaciology, Vol. 14, No. 72, p. 467-77.

Mendelson, M.I. 1969. Average grain size in polycrystalline ceramics. Journal of the American Ceramic Society, Vol. 52, p. 443-46.

Underwood, E.E. 1970. Quantitative stereology. Reading, MA, Addison-Wesley Publishing. 